\title{
Rise and fall, and provisional rebirth of exercise stress testing at the dawn of the third millennium
}

\author{
Mario Petretta, MD, FAHA \\ a Department of Translational Medical Sciences, University Federico II, Naples, Italy
}

Received Nov 13, 2019; accepted Nov 13, 2019

doi: 10.1007/s12350-019-01967-7

\section{See related article, pp. 2056-2066}

The use of electrocardiographic (ECG) changes recorded during exercise stress testing to diagnose ischemic heart disease has a long history. Feil and Siegel in 1928 described changes in ST-segment and T-wave in patients with known angina pectoris during chest pain induced by after repeatedly sitting and standing up, and its regression after cessation of the activity or the administration of nitroglycerine medication. ${ }^{1}$ Subsequently, several authors ${ }^{2-4}$ demonstrated the usefulness of analyzing ECG changes before and after exertion for the detection of myocardial ischemia. In 1956 Robert Bruce $^{5}$ implemented an exercise protocol useful to functionally classify subjects according to New York Heart Association criteria. This protocol initiated the contemporary era of exercise testing, and many of the stress test protocols currently utilized for diagnostic, as well prognostic purpose are based on modifications of those described by Bruce. From the late 1970s, the application of Bayesian Analysis to exercise stress test contributed significantly to improving the accuracy of the test and now many guidelines rely on pre-test likelihood of coronary artery disease (CAD) to select the more appropriate diagnostic test. ${ }^{6-8}$ With the development of radionuclide techniques that permit imaging the myocardium or the left ventricular (LV) blood pool at rest and, most importantly, during exercise, exercise

Reprint requests: Mario Petretta, MD, FAHA, Department of Translational Medical Sciences, University Federico II, Naples, Italy; petretta@unina.it

J Nucl Cardiol 2021;28:2067-71.

1071-3581/\$34.00

Copyright (C) 2019 American Society of Nuclear Cardiology. stress test entered a new era, but significantly increased cost and introduced a radiation burden. ${ }^{9}$

\section{GUIDELINES AND ECG STRESS TESTING FOR ASSESSMENT OF CAD}

Starting from the second half of the 1960s, the ECG stress testing was increasingly used as a non-invasive, cost-effective method to evaluate CAD. However, something has recently changed in the experts' consensus. In fact, the 2013 European Society of Cardiology (ESC) guidelines on stable CAD recommended exercise ECG as the initial test to establish a diagnosis of stable CAD in patients with symptoms of angina and intermediate pre-test likelihood of CAD (15\% to 65\%), free of anti-ischaemic drugs, unless they cannot exercise or display ECG changes that make the ECG nonevaluable. ${ }^{10}$ Similarly, the 2012 American College of Cardiology/American Heart Association guidelines recommended with a Level of Evidence A exercise ECG testing for patients with an intermediate pre-test probability of CAD who have an interpretable ECG and at least moderate physical functioning or no disabling comorbidity. ${ }^{11}$ However, the recent ESC 2019 guidelines downgraded the role of exercise stress test alone and state that "exercise ECG may be considered as an alternative test to rule-in or rule-out CAD only when other non-invasive or invasive imaging are not available," and "exercise ECG is recommended for the assessment of exercise tolerance, symptoms, arrhythmias, BP response, and event risk only in selected patients." 12 The 2019 ESC guidelines also state that it may be beneficial to assess non-invasively for silent ischemia asymptomatic patient every 3 to 5 years, preferably applying stress imaging, while coronary computed tomography angiography should not be used for follow-up of patients with established CAD given the lack of functional information related to ischemia but only for evaluation of CABG patency. ${ }^{12}$ The recent NICE guidelines state that exercise ECG may be used 
instead of functional imaging for subjects with confirmed CAD but not for people without known CAD. ${ }^{13}$

\section{DIAGNOSTIC AND PROGNOSTIC VALUE OF EXERCISE LEVEL}

Beyond ST-segment depression, exercise stress test provides other valuable diagnostic and prognostic information. They include exercise capacity, chronotropic response, heart rate recovery, blood pressure response, and scores such as the Athens QRS score and the Duke Treadmill Score. However, among the various parameters one of the most important is the functional capacity assessed as metabolic equivalents (METs). ${ }^{14}$ A MET is defined as the resting metabolic rate, is $\sim 3.5 \mathrm{~mL} 0_{2} / \mathrm{kg} /$ $\min (1.2 \mathrm{kcal} / \mathrm{minutes}$ for a subject of $70 \mathrm{~kg})$. Despite the reliability of this parameter for assessing the functional capacity has been questioned, ${ }^{15}$ in particular in CAD patients, ${ }^{16}$ the MET concept is a practical, and easily understood procedure for expressing the energy cost of physical activities, to be included in the final report of an exercise stress test.

Previous studies evaluated the added prognostic value of myocardial perfusion imaging (MPI) over exercise ECG stress testing alone in individuals reaching a workload of $\geq 10$ METs. ${ }^{17-21}$ In a first study, Bourque et al. ${ }^{17}$ found that the achievement of $\geq 10$ METs was associated with a very low prevalence of $\geq 10 \% \mathrm{LV}$ ischemia on MPI. No patient (31\% of overall study population) achieving $\geq 85 \%$ of maximal predict heart rate and $\geq 10$ METs without exercise ST-segment depression had this degree of ischemia by SPECT. The Authors concluded a strategy avoiding that further risk stratification with MPI in subjects achieving $\geq 85 \%$ of their maximum age-predicted heart rate and $\geq 10$ METs without ischemic ST-segment depression would be appropriate and cost savings. In a subsequent study, ${ }^{18}$ the same group aimed to prospectively evaluate mortality and non-fatal cardiac events in patients at either intermediate pre-test risk for $\mathrm{CAD}$ or patients with known CAD, achieving $\geq 10$ METs regardless of peak exercise heart rate. These patients had very low rates of cardiac mortality, non-fatal MI, and late revascularization, irrespective of heart rate achieved. Cardiac events did not correlate with abnormalities on the index MPI study. The results suggest that patients who attain $\geq 10$ METS during exercise stress have an excellent prognosis over an intermediate term of follow-up, regardless of peak exercise heart rate achieved, questioning the added value of MPI to standard exercise ECG testing in these subjects. ${ }^{18}$ In diabetic patients, Bourque et al. ${ }^{19}$ found that only $1.8 \%$ of patients able to reach 10 METs of exercise workload had $\mathrm{a} \geq 10 \% \mathrm{LV}$ ischemia. It should be considered that In this study, only 197 of 570 patients
(34.6\%) underwent exercise stress, and during a median 4.4 years follow-up there were only 8 events in the exercise subgroup versus 49 events in the pharmacological stress patients. Thus, exercise variables were not considered in the Cox model. In a further investigation, using two different METs cut-off, Padala et al. ${ }^{20}$ found that, despite significant perfusion defects, diabetic patients who achieve $\geq 5$ METs during stress MPI have a low risk of cardiac events with an annual event rate of $1.1 \%$ person-years and those reaching $\geq 10$ METs have an annualized event rate as low as $0.9 \%$ person-years, respectively. The Authors concluded that exercise level reached during stress MPI is a surrogate for outcomes in diabetic patients undergoing nuclear stress imaging testing. ${ }^{20}$ In this study, non diabetic patients achieving $\geq 5$ METs during stress MPI have a risk of cardiac events of $0.4 \%$ person-years. Interestingly, it has been reported that a substantial proportion of adults $\geq 65$ who undergo exercise SPECT MPI can achieve $\geq 10$ METS and these subjects have low rates of significant LV ischemia and subsequent cardiac events. ${ }^{21}$ However, it should be noted that 5 METs are reached at the first stage and 10 METs at the third stage of a treadmill test with the Bruce protocol, while using a bicycle ergometer 10 METs are difficult to reach for subjects $>80 \mathrm{~kg}$. In fact, considering the relation between METs and watts when exercising on a bicycle ergometer: METs $=(((1.8$ $\times$ watts $\times 6) / \mathrm{kg}+7) / 3.5$, subjects of $90 \mathrm{~kg}$ need to exercise to at least 240 watts to reach 10 METs. ${ }^{14}$

Overall, these results ${ }^{17-21}$ suggest that subjects who achieve $\geq 10$ METs on exercise testing in the absence of signs of stress-induced myocardial ischemia have a good prognosis and that the value and cost-effectiveness of MPI over standard exercise ECG testing in these patients is questionable. Thus a "provisional tracer injection protocol" has been proposed in which a patient would not have the tracer administered intravenously if $\geq 10$ METs was achieved without symptoms and with a negative stress ECG response, ${ }^{22}$ a protocol that warrants further consideration and prospective assessment. $^{23}$

\section{METS AND MPI IN PATIENTS WITH KNOWN CAD}

The incremental prognostic value over ECG stress testing alone specifically in patients with known CAD who achieved $\geq 10$ METs has not been previously investigated. To overcome this shortcoming, in a retrospective study Peclat et al. ${ }^{24}$ evaluated the prognostic value of myocardial perfusion imaging (MPI) in 926 patients with known CAD and high exercise capacity. After the index test, patients were followed for a mean of $32.4 \pm 9.7$ months for the occurrence of a combined 
end-point of all-cause death or non-fatal myocardial infarction (MI). Cardiac workload $<10$ METs and abnormal MPI scan were independent predictors of hard events. The patients achieving $\geq 10$ METs were younger, predominantly male, and had lower prevalence of cardiovascular risk factors and had a lower event rate compared to those achieving $<10$ METs $(1.13 \% / y e a r$ vs $3.95 \% / y e a r, P<.001)$. Interestingly, among patients who achieved $\geq 10$ METs, those with normal scans had a very low rate $(0.57 \%$ /year), lower then those with abnormal scans $(3.37 \%$ year, $P=.023)$. From this study it appears that MPI is able to stratify patients with known CAD achieving $\geq 10$ METs for the occurrence of all-cause death and non-fatal MI. If the results of this study can be generalized to all patients with known CAD it remains to be determined. In fact, the study population investigated by Peclat et al. ${ }^{24}$ seems rather unique, perhaps not representative of all patients with CAD known as a whole, due to the a high prevalence of previous coronary revascularization and, nevertheless, a low incidence of events during follow-up. From Table 1 of Peclat et al. ${ }^{24}$ study it appears that almost all patients (356/384, 93\%) achieving $\geq 10$ METs had previous percutaneous coronary intervention (PCI) vs 253/542 $(47 \%)$ of those achieving $<10$ METs $\left(\chi^{2} 211\right.$, $P<.001)$ : Moreover many of PCI patients also underwent coronary artery bypass graft (CABG). The Authors did not include previous revascularization as a potential predictor of hard events, thus we cannot exclude that this covariate is significantly associate with the clinical outcome. It is also a pity that the authors did not report how much time has elapsed since the last PCI or CABG before performing the stress MPI, in particular in the large subgroup of asymptomatic patients. In fact, it has been demonstrated that, after PCI, MPI results are useful to quantify the event risk and its temporal variation. ${ }^{25}$ On the other hand, MPI screening in the initial post$\mathrm{CABG}$ period is not routinely recommended and current guidelines do not support the use of routine imaging with either exercise or pharmacologic stress testing in asymptomatic patients within 5 years post-CABG. ${ }^{11}$ Also the appropriate use criteria define stress testing early after coronary revascularization as either "rarely appropriate", or "uncertain benefit", while stress MPI performed 5 years after $\mathrm{CABG}$ is considered useful to stratify patients at higher risk of cardiac events. ${ }^{26,27}$

An aspect of this study that deserves consideration is the low prevalence of inducible ischemia and of chest pain during the stress test. Accordingly, not only the presence of ST-segment depression but also the summed difference score were not predictive of events. For the ECG stress test data, this result is in line with the prospective study of the second Medical, Angioplasty, or Surgery Study (MASS II), a randomized clinical trial designed to compare the long term effects of optimal medical therapy, PCI, or CABG in patients with stable multivessel CAD and preserved systolic ventricular function. ${ }^{28}$ In this study, the presence of exertional angina or ST segment depression at 0.08 seconds after the $\mathrm{J}$ point were not associated with the occurrence of cardiovascular events during a 10-year follow-up. However, in the Acampa et al. study ${ }^{25}$ at parametric Weibull survival analysis the presence of inducible ischemia on stress MPI performed 12 to 18 months after the index PCI was associated with an increased, accelerating risk of cardiac events.

In the study of Peclat et al. ${ }^{24}$ the prognostic value of MPI appears to be driven mostly by the presence and, perhaps, extension of scar, despite the lack of predictive value of $\mathrm{LV}$ ejection fraction. The Authors do not mention how many patient had an implantable cardioverter defibrillator or a sudden death, thus it is not known whether the presence of necrosis was in any way related to arrhythmic events. Also the impact of early or late coronary revascularization after the index MPI study was not evaluated, and it has been demonstrated, considering revascularization as a time-dependent covariate, that both the degree of stress-induced ischemia and LV ejection fraction predict the effect of revascularization on outcome in patients with suspected or known CAD. ${ }^{29}$ Finally, the Authors do not clarify if stress MPI was able to further stratify patients achieving $<10$ METs. On the other hand, it is conceivable that other parameters obtainable from stress MPI can provide incremental prognostic information. ${ }^{30-32}$

\section{CONCLUSIONS}

In conclusion, despite its limitations, the study of Peclat et al. $^{24}$ contrasts the hypothesis that an ECG exercise test can be performed alone in patients with good functional capacity and seems to confirm the recommendations of recent guidelines ${ }^{12,13}$ to always associate an imaging test to exercise ECG, even in patients with known CAD.

\section{References}

1. Feil H, Siegel M. Electrocardiographic changes during attacks of angina pectoris. Am J Med Sci 1928;175:225-60.

2. Wood FC, Wolferth CC. Angina pectoris. The clinical and electrocardiographic phenomena of the attack and their comparison with the effects of experimental temporary coronary occlusion. Arch Intern Med 1931;47:339-65. https://doi.org/10.1001/archinte. 1931.00140210002001.

3. Missal ME. Exercise tests and the electrocardiogram in the study of angina pectoris. Ann Int Med 1938;1:201836. https://doi.org/10. 1059/0003-4819-11-11-2018. 
4. Master AM, Jaffe HL. The electrocardiographic changes after exercise in angina pectoris. J Mt Sinai Hosp N Y 1941;7:629-32.

5. Bruce RA. Evaluation of functional capacity and exercise tolerance of cardiac patients. Mod Concepts Cardiovasc Dis 1956;25:321-6.

6. Diamond GA, Forrester JS, Hirsch M, Staniloff HM, Vas R, Berman DS, et al. Application of conditional probability analysis to the clinical diagnosis of coronary artery disease. J Clin Invest 1980;65:1210-21.

7. Froelicher VF, Lehmann KG, Thomas R, Goldman S, Morrison D, Edson R, et al. The electrocardiographic exercise test in a population with reduced workup bias: Diagnostic performance, computerized interpretation, and multivariable prediction. Veterans Affairs Cooperative Study in Health Services \#016 (QUEXTA) Study Group. Quantitative Exercise Testing and Angiography. Ann Intern Med 1998;128:965-74.

8. Chaitman BR. The changing role of the exercise electrocardiogram as a diagnostic and prognostic test for chronic ischemic heart disease. J Am Coll Cardiol 1986;8:1195-210.

9. Montalescot G, Sechtem U, Achenbach S, Andreotti F, Arden C, Budaj A, et al. 2013 ESC guidelines on the management of stable coronary artery disease: The Task Force on the management of stable coronary artery disease of the European Society of Cardiology. Eur Heart J 2013;34:2949-3003. https://doi.org/10.1 093/eurheartj/eht296.

10. Epstein SE. Implications of probability analysis on the strategy used for non-invasive detection of coronary artery disease. Role of single or combined use of exercise electrocardiographic testing, radionuclide cineangiography and myocardial perfusion imaging. Am J Cardiol 1980;46:491-9.

11. Fihn SD, Gardin JM, Abrams J, Berra K, Blankenship JC, Dallas AP, American College of Cardiology Foundation/American Heart Association Task Force, et al. ACCF/AHA/ACP/AATS/PCNA/ SCAI/STS guideline for the diagnosis and management of patients with stable ischemic heart disease: A report of the American College of Cardiology Foundation/American Heart Association task force on practice guidelines, and the American College of Physicians, American Association for Thoracic Surgery, Preventive Cardiovascular Nurses Association, Society for Cardiovascular Angiography and Interventions, and Society of Thoracic Surgeons. Circulation 2012;2012:e354-471. https://doi. org/10.1161/CIR.0b013e318277d6a0.

12. Knuuti J, Wijns W, Saraste A, Capodanno D, Barbato E, FunckBrentano C, et al. ESC Guidelines for the diagnosis and management of chronic coronary syndromes. The Task Force for the diagnosis and management of chronic coronary syndromes of the European Society of Cardiology (ESC). Eur Heart J. 2019. http s://doi.org/10.1093/eurheartj/ehz425.

13. National Institute for Health and Clinical Excellence: Guidance. Chest Pain of Recent Onset: Assessment and Diagnosis of Recent Onset Chest Pain or Discomfort of Suspected Cardiac Origin. National Clinical Guideline Centre for Acute and Chronic Conditions (UK). Royal College of Physicians (UK), London. https:// www.nice.org.uk/donotdo/do-not-use-exercise-electrocardiogramecg-to-diagnose-or-exclude-stable-angina-for-people-without-kno wn-coronary-artery-disease-cad. Accessed 10 Nov 2019.

14. Fletcher GF, Ades PA, Kligfield P, Arena R, Balady GJ, Bittner VA, et al. American Heart Association Exercise, Cardiac Rehabilitation, and Prevention Committee of the Council on Clinical Cardiology, Council on Nutrition, Physical Activity and Metabolism, Council on Cardiovascular and Stroke Nursing, and Council on Epidemiology and Prevention. Exercise standards for testing and training: A scientific statement from the American Heart
Association. Circulation 2013;128:873-934. https://doi.org/10.11 61/cir.0b013e31829b5b44.

15. Byrne NM, Hills AP, Hunter GR, Weinsier RL, Schutz Y. Metabolic equivalent: One size does not fit all. J Appl Physiol 2005;99:1112-9.

16. Savage PD, Toth MJ, Ades PA. A re-examination of the metabolic equivalent concept in individuals with coronary heart disease. $\mathrm{J}$ Cardiopulm Rehabil Prev 2007;27:143-8.

17. Bourque JM, Holland BH, Watson DD, Beller GA. Achieving an exercise workload of $\geq 10$ metabolic equivalents predicts a very low risk of inducible ischemia: Does myocardial perfusion imaging have a role? J Am Coll Cardiol 2009;54:538-45. https://doi. org/10.1016/j.jacc.2009.04.042.

18. Bourque JM, Charlton GT, Holland BH, Belyea CM, Watson DD, Beller GA. Prognosis in patients achieving $\geq 10$ METS on exercise stress testing: Was SPECT imaging useful? J Nucl Cardiol 2011;18:230-7. https://doi.org/10.1007/s12350-010-9323-2.

19. Bourque JM, Patel CA, Ali MM, Perez M, Watson DD, Beller GA. Prevalence and predictors of ischemia and outcomes in outpatients with diabetes mellitus referred for single-photon emission computed tomography myocardial perfusion imaging. Circ Cardiovasc Imaging 2013;6:466-77. https://doi.org/10.1161/CIRCIMAGING. 112.000259 .

20. Padala SK, Ghatak A, Padala S, Katten DM, Polk DM, Heller GV. Cardiovascular risk stratification in diabetic patients following stress single-photon emission-computed tomography myocardial perfusion imaging: The impact of achieved exercise level. J Nucl Cardiol 2014;21:1132-43. https://doi.org/10.1007/s12350-014-99 86-1.

21. Smith L, Myc L, Watson D, Beller GA, Bourque JM. A high exercise workload of $\geq 10$ METS predicts a low risk of significant ischemia and cardiac events in older adults. J Nucl Cardiol. 2018. https://doi.org/10.1007/s12350-018-1376-7.

22. Duvall WL, Levine EJ, Moonthungal S, Fardanesh M, Croft LB, Henzlova MJ. A hypothetical protocol for the provisional use of perfusion imaging with exercise stress testing. J Nucl Cardiol 2013;20:739-47. https://doi.org/10.1007/s12350-013-9710-6.

23. Beller GA, Bateman TM. Provisional use of myocardial perfusion imaging in patients undergoing exercise stress testing: A worthy concept fraught with challenges. J Nucl Cardiol 2013;20:711-4. h ttps://doi.org/10.1007/s12350-013-9742-y.

24. Peclat T, do Amaral Henrique de Souza V, Freitas Souza V, Mitiko Koda Nakamoto A, Martins Neves F, Silva I, et al. The additional prognostic value of myocardial perfusion SPECT in patients with known coronary artery disease with high exercise capacity. J Nucl Cardiol (in press).

25. Acampa W, Evangelista L, Petretta M, Liuzzi R, Cuocolo A. Usefulness of stress cardiac single-photon emission computed tomographic imaging late after percutaneous coronary intervention for assessing cardiac events and time to such events. Am J Cardiol 2007;100:436-41.

26. Hendel R, Berman D, Di Carli M, Heidenreich P, Henkin R, Pellikka P. Appropriate use criteria for cardiac radionuclide imaging: A report of the American College of Cardiology Foundation Appropriate Use Criteria Task Force, the American Society of Nuclear Cardiology, the American College of Radiology, the American Heart Association, the American Society of Echocardiography, the Society of Cardiovascular Computed Tomography, the Society for Cardiovascular Magnetic Resonance, and the Society of Nuclear Medicine. J Am Coll Cardiol 2009;53:2201-29.

27. Acampa W, Petretta M, Evangelista L, Nappi G, Luongo L, Petretta MP, Cuocolo A. Stress cardiac single-photon emission computed tomographic imaging late after coronary artery bypass surgery for risk stratification and estimation of time to cardiac 
events. J Thorac Cardiovasc Surg 2008;136:46-51. https://doi.org/ 10.1016/j.jtcvs.2007.10.011.

28. Garzillo CL, Hueb W, Gersh B, Rezende PC, Lima EG, Favarato $\mathrm{D}$, et al. Association between stress testing-induced myocardial ischemia and clinical events in patients with multivessel coronary artery disease. JAMA Intern Med. 2019. https://doi.org/10.1001/ja mainternmed.2019.2227.

29. Petretta M, Acampa W, Daniele S, Zampella E, Assante R, Nappi $\mathrm{C}$, et al. Long-term survival benefit of coronary revascularization in patients undergoing stress myocardial perfusion imaging. Circ J 2016;80:485-93. https://doi.org/10.1253/circj.CJ-15-1093.

30. Petretta M, Storto G, Acampa W, Sansone V, Evangelista L, Spinelli L, et al. Relation between wall thickening on gated perfusion SPECT and functional recovery after coronary revascularization in patients with previous myocardial infarction. Eur J Nucl Med Mol Imaging 2004;31:1599-605.

31. Petretta M, Acampa W, Daniele S, Petretta MP, Plaitano M, Cuocolo A. Transient ischemic dilation in patients with diabetes mellitus: Prognostic value and effect on clinical outcome after coronary revascularization. Circ Cardiovasc Imaging 2013;6:90815. https://doi.org/10.1161/CIRCIMAGING.113.000497.

32. Slomka P. Leveraging latest computer science tools to advance nuclear cardiology. J Nucl Cardiol 2019;26:1501-4. https://doi.or g/10.1007/s12350-019-01873-y.

Publisher's Note Springer Nature remains neutral with regard to jurisdictional claims in published maps and institutional affiliations. 